\title{
Nefoussa (Djebel) : Géographie, Histoire et Société
}

\author{
Jean-Louis Ballais et Joseph Taïeb
}

\section{OpenEdition}

Journals

Édition électronique

URL : https://journals.openedition.org/encyclopedieberbere/2696

DOI : $10.4000 /$ encyclopedieberbere. 2696

ISSN : 2262-7197

\section{Éditeur}

Peeters Publishers

\section{Édition imprimée}

Date de publication : 4 octobre 2012

Pagination : 5353-5359

ISBN : 978-90-429-2640-0

ISSN : 1015-7344

\section{Référence électronique}

Jean-Louis Ballais et Joseph Taïeb, « Nefoussa (Djebel) : Géographie, Histoire et Société »,

Encyclopédie berbère [En ligne], 33 | 2012, document N26, mis en ligne le 15 mars 2021, consulté le 17 février 2022. URL : http://journals.openedition.org/encyclopedieberbere/2696 ; DOI : https://doi.org/ 10.4000/encyclopedieberbere.2696

Ce document a été généré automatiquement le 17 février 2022.

(c) Tous droits réservés 


\title{
Nefoussa (Djebel) : Géographie, Histoire et Société
}

\author{
Jean-Louis Ballais et Joseph Taïeb
}

\section{Géographie Physique}

1 À la latitude du Mzab et de Ouargla, le djebel Nefoussa constitue la prolongation, en Libye, des Monts de Matmata* - djebel Dammar - djebel Abiod (Taïeb, Ballais 2010) où s'amorce une modification de direction à environ $90^{\circ}$ qui oriente le relief jusqu'au rivage méditerranéen, à l'Est de Tripoli. Il se caractérise donc comme un relief long de 190 kilomètres (entre la frontière tunisienne et Gharyan), étroit (une vingtaine de kilomètres), haut de 600 à $800 \mathrm{~m}$, dissymétrique, avec un talus abrupt, au nord, qui domine la plaine de la Djeffara et, au contraire, une pente douce (Guibla) vers le sud, en direction du Sahara (Hamada el-Homra). Le relief et le modelé de cette montagne demeurent peu connus, tout particulièrement dans les sources en langue française.

Le relief serait une cuesta, vestige d'un grand bombement à axe Nord-Ouest/Sud-Est centré sur la Djeffara actuelle, dont la corniche est constituée de calcaires turoniens (calcaire de Nalout) au-dessus de formations marneuses du Crétacé, le tout avec un pendage très faible vers le sud. Des formations plus récentes (Crétacé supérieur) et de résistance inégale accidentent le revers ou Dahar (Despois 1935).

Mais la principale originalité, comme dans les Monts de Matmata, est constituée par une formation superficielle héritée, la terre hamri qui recouvre indifféremment toutes les roches du substratum. D'abord interprétée comme une formation loessique sableuse (Pfalz 1929 ; Despois 1935), de nouvelles analyses (Assallay et al. 1996) ont montré qu'il s'agit de loess typique. C'est cette formation superficielle qui, quand elle est assez épaisse, permet la mise en culture du djebel, surtout dans la partie orientale où les précipitations croissent (elles dépassent 300 à $350 \mathrm{~mm} / \mathrm{an}$ alors qu'elles se réduisent à moins de $150 \mathrm{~mm}$ à la frontière tunisienne) grâce à l'augmentation de l'altitude et à la réduction de la distance au littoral méditerranéen. 


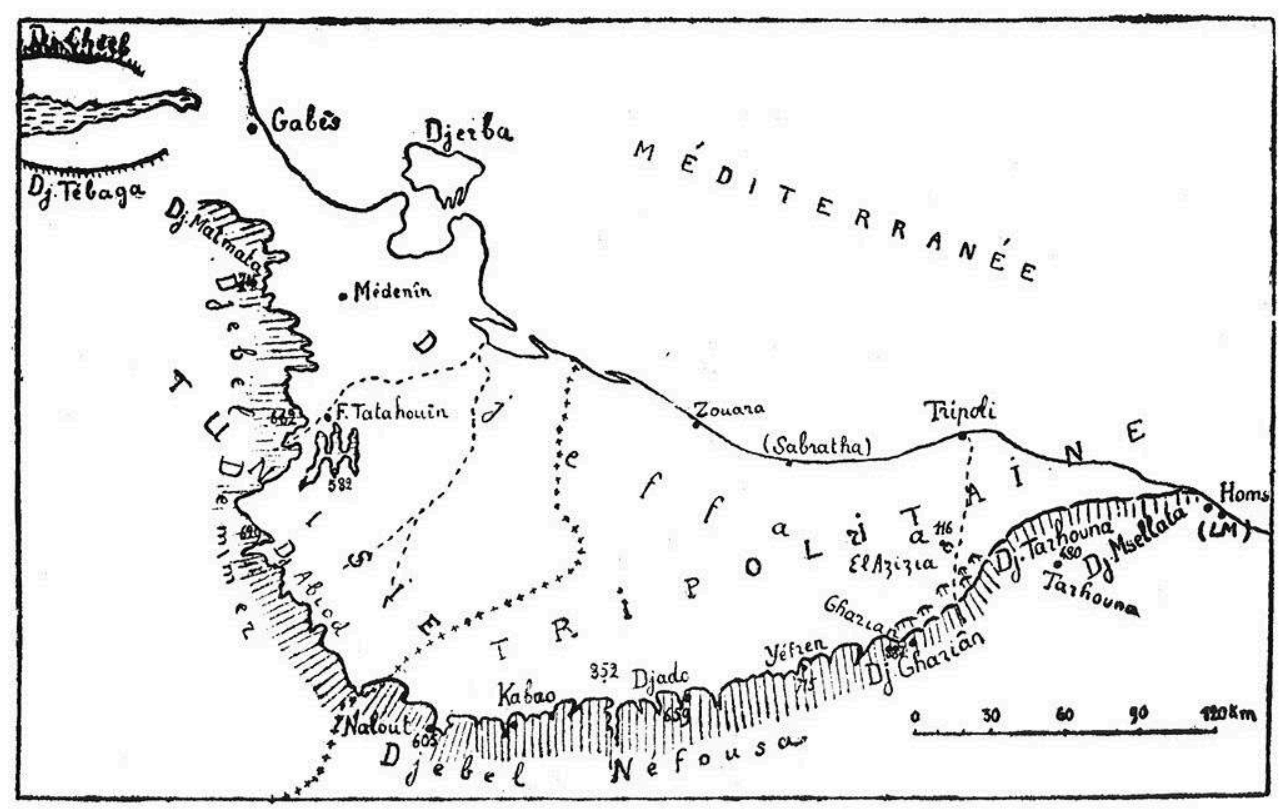

Fig. 1. CARTE SOMmaire dU DJebel Nefoussa (DESPOIS 1935, P. 2).

\section{Géographie humaine, Histoire et Société}

4 Le Djebel Nefoussa (Nafûsa en arabe) adrar $n$ infusen en berbère, fait référence à une population berbérophone installée à date ancienne, puisque Ibn Khaldoun les mentionne, au XIV siècle.

\section{Le milieu physique et la vie agraire}

5 Il s'agit d'un escarpement rocheux, au sud-ouest de Tripoli, dominant la plaine alluviale de la Djeffara, prolongement oriental de la Djeffara tunisienne. Etendu d'ouest en est sur quelque $200 \mathrm{~km}$, le djebel est à plus de $100 \mathrm{~km}$ de la mer, en moyenne $150 \mathrm{~km}$ dans la partie occidentale (Despois 1935).

D'orientation sud-ouest-nord-est, le djebel est exposé largement au vent frais et humide du nord-ouest, le gharbî en arabe, avec des précipitations annuelles dépassant $300 \mathrm{~mm}$ en certains lieux. Le paysage est donc plus vert et plus riche que dans les djebels tunisiens, avec un retour en force des essences méditerranéennes comme le thym, le romarin, le câprier sauvage et la merrûbiyya, plante médicinale, peut-être l'équivalent de la simarùba corse et italienne (Despois 1935).

7 Vers l'Est, le Nefoussa se prolonge par le djebel Gharyân aux conditions physiques peu différentes. Il faut aussi noter que des sources existent au contact des calcaires durs et perméables des horizons supérieurs et des couches plus tendres et plus perméables des horizons inférieurs des reliefs de côtes. Fonds de vallée, ravins, etc., paraissent surdimensionnés dans ce climat relativement sec où les cours d'eau ne sont pas pérennes. Sans doute comme dans les djebels du sud tunisien s'agit-il d'une morphologie façonnée par des paléoclimats plus humides.

8 Comme dans les djebels du sud tunisien voisin, les populations exploitent les potentialités des trois éléments morphologiques de la région. Sur les talus du djebel 
proprement dits alternent plantations d'oliviers, petits champs de tabac, abricotiers, figuiers, amandiers. Sur le Dahar (dahr, dos en arabe), c'est-à-dire le plateau saharien, sont envoyés, avec quelques bergers, les troupeaux d'ovins. La Djeffara, enfin, est terre de labour et accessoirement de pâturage, mais seule une fraction de la population se déplace sous la tente pour accompagner les moutons, labourer et moissonner.

L'ensemble de la population est sédentaire et les déplacements hors du djebel, pour ceux qui accompagnent les troupeaux, ne dépassent pas quatre mois par an. Nous avons donc affaire à des sédentaires transhumants plutôt qu'à des semi-nomades (Despois 1935). Comme en Tunisie, l'habitat de ces montagnards est en grande partie troglodyte et là, particularité du djebel, beaucoup de villages sont restés sur les hauteurs des éperons calcaires, donc sur des sites défensifs, alors que la plupart de ces sites ont été abandonnés en Tunisie au cours des trois derniers siècles. La modicité des ressources, en dépit d'une pluviométrie moins chiche que dans le sud tunisien, pousse depuis des générations beaucoup de ruraux à migrer vers les villes de la côte tripolitaine, et plus encore vers Tunis où ils exercent de petits métiers.

10 Ce tableau de la vie traditionnelle était parfaitement vérifié jusqu'au début des années 1950. Depuis, il s'est modifié sans toutefois disparaître et il serait intéressant, dans l'étude du milieu humain, d'analyser ces transformations.

\section{Le milieu humain et le contexte historique}

11 Le djebel a la particularité de compter, au plan linguistique, deux populations: la berbérophone et l'arabophone, ainsi que deux entités confessionnelles: le sunnismemalékite, et l'ibadisme (Agabi 2000), ibadisme et berbérité se recoupant presque exactement. Il y a une grosse centaine d'agglomérations dans le Nefoussa, petites villes, bourgades, hameaux et surtout villages, installées sur les festons des reliefs de côtes, pour la plupart, et dominant la Djeffara. D'autres, en revanche, occupent des sites plus en arrière sur les plateaux comme Nâlût à l'extrémité ouest du djebel, Chrûs ou Srûs un peu plus à l'est, Fâssatû et Rahibât au centre. Très rares sont en revanche les sites de piémont à l'orée de la Djeffara, au pied du djebel comme par exemple Chaqchûq (Goldberg 1981).

12 Nâlût est dans le massif l'agglomération la plus occidentale, Qiqla et Al-Guel'A les agglomérations les plus orientales (Goldberg 1981). La population berbéro-ibadite occupe quatre blocs de villages séparés par des espaces arabo-sunnites. Le premier bloc, celui de Nâlût-Wazzân, le plus à l'ouest, ne compte que quelques villages. Plus à l'est, le deuxième bloc, celui de Kabao, est plus conséquent, avec six à sept villages. Le troisième est le plus important: il a comme centre Fâssâtû et comprend une petite ville très ancienne, Jâdû ; toujours au centre du djebel, mais plus décalé vers l'est, le quatrième et dernier bloc, lui aussi berbérophone, autour d'Yfren, compte plus de douze villages dans la partie centre-est du massif. L'extrémité est, en revanche, ne comporte aucun village berbérophone. Ce sont donc les parties ouest et centrale du djebel qui sont ibadites et berbérophones (de Planhol 1997).

Le premier bloc est séparé du deuxième par au moins cinq villages arabo-phones et sunnites. Le deuxième est séparé du troisième par cinq ou six villages toujours arabophones et sunnites mais aussi, fait intéressant, par huit, voire plus, villages mixtes, configuration originale avec deux quartiers séparés religieusement et linguistiquement. Peut-être cette séparation des villages en deux entités s'explique-t- 
elle par le fait qu'un des clans du village passait au sunnisme et à l'arabe tandis que l'autre restait fidèle à la tradition berbère et ibadite. Le troisième bloc est séparé du quatrième par une vingtaine de villages, entièrement arabophones et sunnites. L'est du djebel enfin compte une dizaine d'agglomérations également arabophones et sunnites (Despois 1935 et 1997). Il faut noter, empiriquement au moins, qu'à l'intérieur du djebel le passage au sunnisme précédait le changement de langue comme si le berbère perdait toute signification avec la disparition de l'ibadisme. Le mouvement de conversion et de changement de langue est relativement ancien et date des $\mathrm{XVII}^{\mathrm{e}}$ et $\mathrm{XVIII}^{\mathrm{e}}$ siècles. Quelques villages, cependant, à l'extrémité est du massif, n'ont été convertis que récemment (XIX ${ }^{\mathrm{e}} \mathrm{XX}{ }^{\mathrm{e}}$ siècles), peut-être en rapport avec le prosélytisme de la confrérie de Sanusiyya, fondée en 1843 en Cyrénaïque, qui est fort influente. Mais, conversion récente ou ancienne au sunnisme, passage à l'arabe séculaire ou rapproché dans le temps, on se souvient partout d'avoir été jadis ibadite et berbérophone.

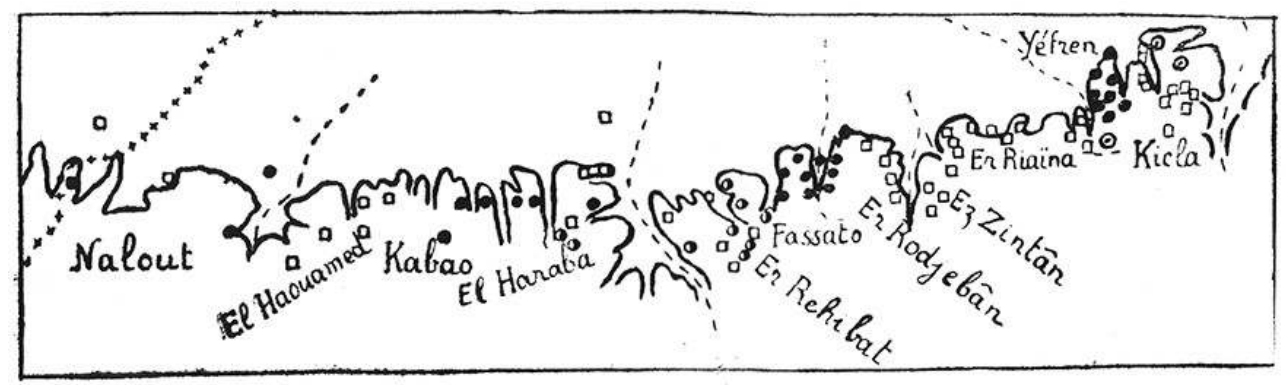

FIC. 9. - Berberophones et Arabophones du Djebel Nefousa

Les ronds noirs désignent les villages berbérophones et les carrés blancs les villages arabophones. Les ronds noirs et blancs indlement des villages mixtes et les ronds blanes point noir les villages, qui, autrefois berberophones, sont arabophones depuis moin de deux siècles.

Fig. 2. Carte des langues (Berbère/ARABe) dans le dJebel nefoussa (Despois 1935, P. 144).

L'originalité du djebel est dans une large mesure un produit de l'histoire. L'arrivée des tribus arabes, les Banû Hillâl et surtout les Banû Sulaym, au milieu du $\mathrm{XI}^{\mathrm{e}}$ siècle, entraîna le repli des Berbères de la Djeffara vers la montagne. Nous avons donc affaire ici à ce que Xavier de Planhol (1968) appelle une montagne refuge. Mais, comme dans les Matmata, à partir du XV siècle ou du $\mathrm{XVI}^{\mathrm{e}}$ siècle, la confédération berbère des Ouerghamma entama une reconquête de la Djeffara absorbant des Arabes et se laissant arabiser par eux. Puissante entité guerrière semi-nomade, les Ouerghamma imposèrent aux montagnards une protection-vassalité qui dura jusqu'au XIX ${ }^{e}$ siècle, tout en se heurtant dans la plaine à la rivalité des Mḥâmîd, probablement de souche arabe. Mais comme rien n'est simple en la matière, le djebel est, à petite échelle, une montagne bédouinisée selon la terminologie de Xavier de Planhol (1968), car les nomades Zîntan ont planté leurs tentes sur le plateau saharien au sud-ouest d'Yfren.

Plus ou moins protégés par les Ouerghamma, les populations du djebel demeurèrent rétives au pouvoir de Tripoli, qu'il ait été celui des pachas autonomes vis-à-vis de la Porte ou celui des gouverneurs ottomans après la reconquête de la Libye par les Turcs en 1835-1860. Il est probable toutefois que la présence d'un pouvoir sunnite dans la capitale ait été un facteur de retour à l'orthodoxie.

Lors de la conquête turque, animée par un chef énergique, Ghûma, la montagne s'opposa vingt-trois ans durant aux troupes de la Porte. Il semble que Ghûma n'ait pas été un montagnard mais un bédouin de la tribu des Mḥâmîd. 
population juive. Certes, la judaïsation massive des Berbères avant l'islamisation du
djebel ne paraît plus aujourd'hui soutenable (Talbi 1971), mais Ibn Khaldoûn signale, malgré tout, des passages au judaïsme à des époques très reculées (Ibn Khaldoûn 1986), passages sans doute individuels chez Jrawa (Igerwan) des Aurès, les Madyuna de l'ouest algérien et les Nefoussi. Autour de l'an mil, le voyageur arabe El-Bekrî confirme la présence de nombreux juifs à Jâdû. À l'époque, le djebel était totalement berbérophone. Les Juifs ne pouvaient donc parler que berbère (El-Bekrî 1965). Or, au XIX ${ }^{\mathrm{e}}$ siècle, ils paraissent bien utiliser l'arabe entre eux et le berbère avec les Berbères. Peut-être furent-ils arabisés lors des persécutions almohades dans la seconde moitié du XII ${ }^{\mathrm{e}}$ siècle, lorsque des Juifs des villes de langue arabe vinrent, vraisemblablement, s'y réfugier. Peut-être l'arabisation fut-elle plus tardive, lorsque les Juifs de Tripoli fuirent la présence espagnole puis celle des chevaliers Hospitaliers de 1510 à 1551 (Mac Lachlan 1993).

Depuis un demi-siècle, la situation dans certains domaines a changé. L'exploitation pétrolière a considérablement enrichi la population libyenne; mais le djebel a peu profité de cette manne et beaucoup d'habitants ont émigré vers les villes de la côte en quête d'opportunités économiques, s'arabisant lentement et optant pour l'orthodoxie sunnite. D'autre part, la jamâhîriyya libyenne s'est voulu, avant tout, état des masses. Or, l'originalité linguistique et religieuse de la montagne résiste à l'uniformisation que cela implique. D'où de la part des autorités de Tripoli, des mesures répressives: interdiction d'écrire le berbère, loi 24-1994 qui interdit de porter des prénoms berbères (Ferreïra 2006).

\section{BIBLIOGRAPHIE}

GÉOGRAPHIE PHYSIQUE

ASSALlay A. M., ROGERS C.D.F. et SMALLEy I. J., 1996 - "Engineering properties of loess in Libya", Journal of Arid Environments, vol. 32, nº 4, p. 373-386.

DESPOIS J., 1935 - Le Djebel Nefousa (Tripolitaine). Étude géographique, Paris, Larose, 349 p.

PFALZ H., 1929 - « Landeskundliche Hauptprobleme Tripolitaniens », Petermanns Mitteilungen, LXXV, n 5 et 6.

TAÏEB J., BALLAIS J.-L., 2010 - « Matmata (Djebel) », Encyclopédie Berbère, XXX, p. 4684-4696, Louvain, Peeters. 
GÉOGRAPHIE HUMAINE

AGABI C., «Ibadites », Encyclopédie berbère, XXIII, 2000, p. 3567-3574.

DESPOIS J., Le djebel Nefoussa (Tripolitaine). Etude géographique, Paris, Larose, 1935 [passim, p. 166 et sq.].

EL-BEKRI Abou Obeïd, Description de l'Afrique septentrionale, traduit de l'arabe par Marc Guckin de Slane, Paris, Adrien Maisonneuve, 1965 [p. 9 du texte arabe joint à la traduction].

GOLDBERG H., Qôrôt Lûv uyéhudéah (en hébreu) [= Histoire de la Libye et de ses Juifs], Jérusalem, Institut Ben Zvi, 1981 [p. 392].

IBN KHALDOUN, Peuples et nations du monde, extraits des 'ibar, traduits de l'arabe et présentés par Abdesselam Cheddadi, Paris, Sindbad (La Bibliothèque arabe), 1986 [p. 490].

MAC LACHLAN K.S., « Nafûsa », Encyclopédie de l'Islam, t. VII, New York-Leïden, E. J. Brill / Paris Maisonneuve et Larose, 1993, p. 895-896.

PLANHOL (de) X., Les fondements géographiques de l'histoire de l'Islam, Paris, Flammarion, 1968

[p. 143-146 et p. 142-143].

PLANHOL (de) X., Minorités en Islam, géographie politique et sociale, Paris, Flammarion (Géographies), 1997 [p. 105].

TALBI M., « Un nouveau fragment de l'histoire de l'Occident musulman : 62-196/682-812, l'épopée d'Al Kahina », Les Cahiers de Tunisie, 19, 1971, p. 19-52.

INDEX

Mots-clés : Géographie, Libye, Nefoussa 